\title{
Energy expenditure during antiorthostatic bed rest (simulated microgravity)
}

\author{
RANDALL J. GRETEBECK, DALE A. SCHOELLER, EVERETT K. GIBSON, \\ AND HELEN W. LANE \\ Nutritional Biochemistry and Stable Isotope Laboratories, Space and Life Sciences Directorate, \\ National Aeronautics and Space Administration, Johnson Space Center, Houston, Texas 77058; \\ and University of Chicago, Department of Medicine, Chicago, Illinois 60637
}

\begin{abstract}
Gretebeck, Randall J., Dale A. Schoeller, Everett K. Gibson, and Helen W. Lane. Energy expenditure during antiorthostatic bed rest (simulated microgravity). J. Appl. Physiol. 78(6): 2207-2211, 1995.-Few studies have addressed the interaction between energy balance and lean body mass in healthy subjects during spaceflight or its simulations. We used doubly labeled water to measure total energy expenditure (TEE) in nine healthy adult men during two 7-day periods, once before and once during a 10-day headdown bed-rest period. Mean TEE was $21 \%$ less during than before bed rest; however, neither basal (BEE) nor resting (REE) energy expenditures changed, implying that the lesser TEE resulted from a reduction in physical activity. During the bed-rest period, energy intake was $563 \pm 280 \mathrm{kcal} / \mathrm{day}$ higher than TEE $(P<0.05)$ but body weight, fluid balance, $\mathrm{BEE}$, and REE did not change relative to before bed rest. However, the small but statistically significant increase in body fat $(0.44 \pm 0.67 \mathrm{~kg}, P<0.05)$ during the bed-rest period suggests that body weight alone does not accurately reflect changes in energy balance during antiorthostatic bed rest.
\end{abstract}

doubly labeled water; basal energy expenditure; resting energy expenditure; sedentary

EXPOSURE TO MICROGRAVITY induces physiological and biochemical changes in humans, including fluid loss, reduced energy intake, and negative nitrogen balance (13). These physiological changes in turn affect body weight, muscle strength, and fatigability and thus can interfere with health and normal functioning. Another less-recognized factor that affects all of these changes is the ongoing loss of body weight due to negative energy balance in space $(1,12,15)$. Ultimately, the combination of these conditions may limit the ability to function at peak levels in the demanding spaceflight environment.

The absence of gravitational forces in space eliminates an important stimulus for maintaining muscular strength and endurance and has been hypothesized to reduce the energy required by humans in spaceflight ( 7 , 12). However, the hypothesis that energy requirements are reduced during spaceflight has not been supported by studies of human energy metabolism during flight. That is, metabolic balance studies, diet histories, records of the disappearance of food, and measurements of respiratory gas production all have suggested that energy requirements during spaceflight are similar to those on Earth (11). Because access to true microgravity (possible only in space) is limited, methods that simulate the physiological effects of spaceflight are often used for ground-based research. One such model often used to study the musculoskeletal changes associ- ated with microgravity is $-6^{\circ}$ head-down tilt (antiorthostatic) bed rest. Even though energy balance has a direct effect on protein metabolism and lean body mass, few studies have assessed the energy requirements of healthy subjects during simulated microgravity. Krebs et al. (9) showed that the body weights of subjects who consumed a constant diet (average 2,592 kcal/day) for 6 wk before and 5 wk during bed rest did not change. However, body composition did change during bed rest: lean body mass decreased by $2.25 \pm 1.88 \mathrm{~kg}$ and body fat increased by $2.70 \pm 1.78 \mathrm{~kg}$. These results suggest that body weight does not accurately reflect energy balance during bed rest and that a diet that maintains body weight in healthy subjects during bed rest may be hypercaloric, since the energy content of body fat is much higher than that of lean body mass.

Understanding how basic energy requirements contribute to overall energy needs during spaceflight and simulations thereof is important to establish appropriate guidelines for energy intake and exercise during and after spaceflight. The purpose of this investigation was to test the hypothesis that energy requirements are reduced during simulated microgravity $\left(-6^{\circ}\right.$ headdown tilt bed rest) and to determine the magnitude of this reduction. To do so, we measured total energy expenditure (TEE) daily in free-living ambulatory subjects and in the same subjects during a 10-day period of simulated microgravity $\left(-6^{\circ}\right.$ head-down tilt bed rest). We also investigated potential differences in energy intake, resting (REE) and basal (BEE) energy expenditures, fluid balance, and body composition before and during bed rest.

\section{METHODS}

Subjects. Nine healthy adult men (mean age $35.8 \pm 4.6 \mathrm{yr}$, height $182 \pm 5.4 \mathrm{~cm}$, and initial weight $84.2 \pm 9.9 \mathrm{~kg}$ ) served as subjects in this study. All signed a statement of informed consent approved by the Johnson Space Center Human Research Policy and Procedures Committee and passed an Air Force Class III physical examination before being admitted to the study.

Protocol. The experimental design is shown in Fig. 1. Briefly, daily energy expenditure was assessed during two 7day periods that were separated by $\geq 60$ days. The first period (ambulatory) was a free-living condition, and the second spanned the 3rd-10th days of a microgravity simulation. This simulation involved confining subjects to hospital beds propped with blocks at the foot to achieve a $-6^{\circ}$ head-down tilt. The tilt was verified with an inclinometer, and subjects were supervised to ensure that they remained in bed at all times during the 10-day study period. Energy expenditure was assessed with doubly labeled water (see below). Body 


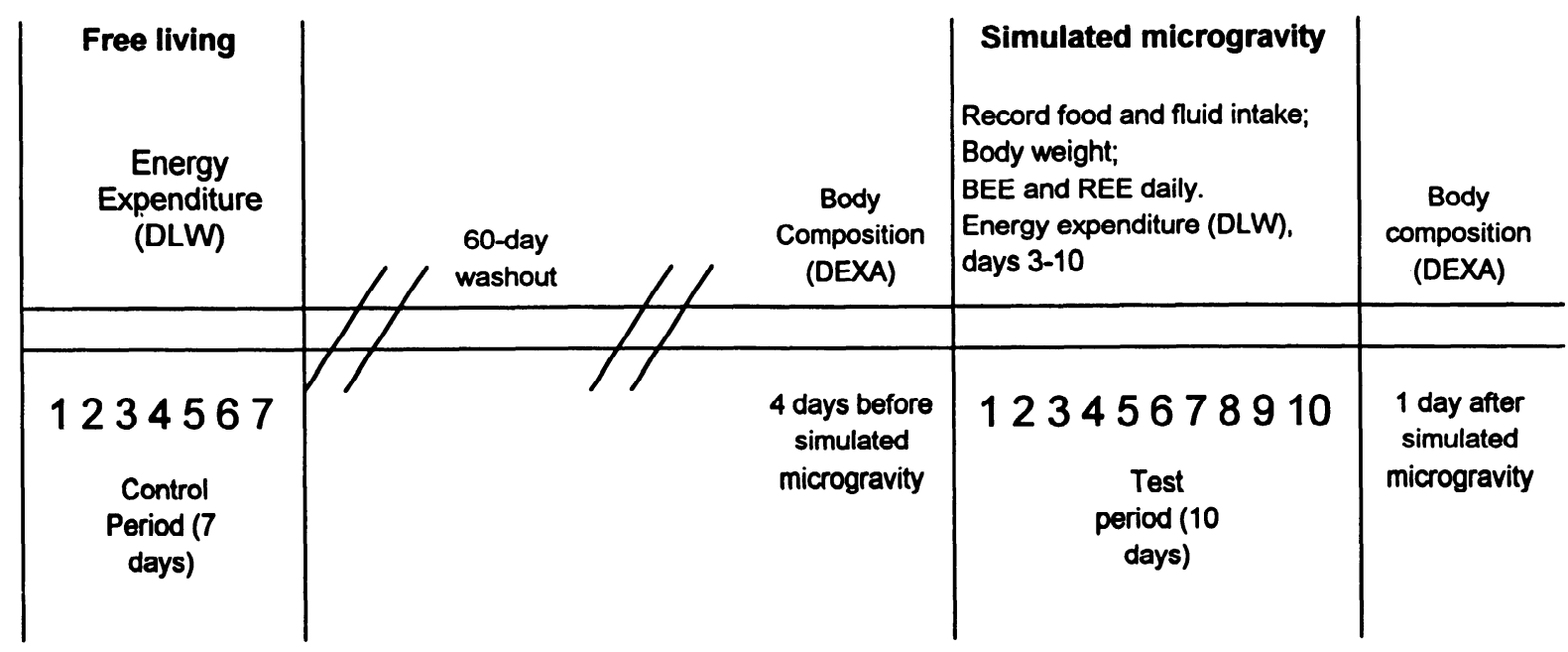

FIG. 1. Diagram of testing timeline. DLW, doubly labeled water; DEXA, dual-energy X-ray absorptiometry; $\mathrm{BEE}$, basal energy expenditure; REE, resting energy expenditure.

composition was measured by dual-energy X-ray absorptiometry once 4 days before the bed-rest period and once the day after the bed-rest period. Subjects selected their own diets during the bed-rest period from a hospital menu. However, subjects were encouraged to eat in such a way as to maintain their body weight during the bed-rest period. Each food item was placed on a separate plate and weighed on an electronic scale by the study investigators before and after each meal. Fluid intake, including water, was measured by volume. The Nutritionist III program (version 7.0, 1991) from N-Squared Computing Analytic Software (Salem, OR) was used to analyze the nutrient and water content of all foods consumed. All urine produced during the bed-rest period was measured by volume. Body weight was measured daily during the bed-rest period.

$T E E$. For the ambulatory control period, subjects reported to the Nutritional Biochemistry Laboratory at Johnson Space Center on the morning of day 1 after having fasted overnight. After providing baseline urine and saliva samples, they then consumed doubly labeled water $\left[10 \% \mathrm{H}_{2}{ }^{18} \mathrm{O}\right.$ (Cambridge Isotope Laboratories, Cambridge, MA) mixed with $99.8 \%{ }^{2} \mathrm{H}_{2} \mathrm{O}$ (Icon Services, Summit, NJ)]. Doses were calculated for each subject to be $0.25 \mathrm{~g}$ of $\mathrm{H}_{2}{ }^{18} \mathrm{O}$ and $0.12 \mathrm{~g}$ of ${ }^{2} \mathrm{H}_{2} \mathrm{O}$ per kilogram of total body water as estimated from body weight. Urine and saliva samples were collected at the laboratory $5 \mathrm{~h}$ after dosing; subjects were instructed to collect their first-morning voids for the ensuing 6 days and deliver them each morning to the laboratory.

During the simulated-microgravity period, subjects were confined to bed at the Clear Lake Regional Medical Center for 10 days, and study investigators collected all samples. All samples were stored frozen in cryogenically stable tubes at $-20^{\circ} \mathrm{C}$ until analysis by isotope-ratio mass spectrometry at Johnson Space Center.

Samples were analyzed in triplicate for ${ }^{2} \mathrm{H}_{2} \mathrm{O}$ by zinc reduction (19). This method involved isolating $2 \mu \mathrm{l}$ of sample in a microcap with zinc reagent under vacuum in break-seal reaction vessels. These vessels were heated in a $540^{\circ} \mathrm{C}$ oven for 30 min shortly before being analyzed. Samples were analyzed for $\mathrm{H}_{2}{ }^{18} \mathrm{O}$ by $\mathrm{CO}_{2}$ equilibration (16), which involved dispensing $1.5 \mathrm{ml}$ of sample into a $7-\mathrm{ml}$ vacutainer tube, filling the tube with $99.9 \%$ pure $\mathrm{CO}_{2}$, and shaking the capped tubes in a water bath at $25^{\circ} \mathrm{C}$ for $\geq 12 \mathrm{~h}$. $\mathrm{CO}_{2}$ was then removed cryogenically and stored in break-seal tubes. Samples were analyzed on a Finnigan MAT 251 stable-isotope mass spectrometer at Johnson Space Center. The reproducibility of this method in this laboratory is generally better than $\pm 0.05 \%$ at the 1 -s confidence level (18).

Dilution spaces for $\mathrm{H}_{2}{ }^{18} \mathrm{O}$ and ${ }^{2} \mathrm{H}_{2} \mathrm{O}$ (in mol) were calculated from the baseline and 5-h samples with $\mathrm{N}=(\mathrm{WA} / 18.02 \mathrm{a})\left[\left(\mathrm{d}_{\mathrm{a}}\right.\right.$ $\left.\left.-d_{t}\right) /\left(d_{s}-d_{p}\right)\right]$, where $N$ is the dilution space; $W$ is the amount of water (in $g$ ) used to dilute the dose; $A$ is the amount of dose (in g) administered; $a$ is the dose (in g) diluted for analysis; and $d$ is the \%o enrichment of a, tap water ( $t$ ), sample after dose (s), or sample before dose (p).

$\mathrm{CO}_{2}$ production rate (in mol/day) was calculated as described by the study of Schoeller et al. (17) with $\mathrm{rCO}_{2}=$ $(\mathrm{N} / 2.078)\left(1.01 \mathrm{k}_{\mathrm{O}}-1.04 \mathrm{k}_{\mathrm{H}}\right)-0.0246 \mathrm{r}_{\mathrm{Gf}}$, where $\mathrm{N}$ is average dilution space from $\mathrm{H}_{2}{ }^{18} \mathrm{O}$ and ${ }^{2} \mathrm{H}_{2} \mathrm{O} ; \mathrm{k}_{\mathrm{O}}$ and $\mathrm{k}_{\mathrm{H}}$ are the turnover rates of $\mathrm{H}_{2}{ }^{18} \mathrm{O}$ and ${ }^{2} \mathrm{H}_{2} \mathrm{O}$, respectively; and $\mathrm{r}_{\mathrm{Gf}}$ is estimated as $1.05 \mathrm{~N}\left(1.01 \mathrm{k}_{\mathrm{O}}-1.04 \mathrm{~K}_{\mathrm{II}}\right)$.

Oxygen consumption was derived by dividing the $\mathrm{CO}_{2}$ production rate by a food quotient representing a typical American diet (for the free-living period) or individually from actual diet composition (for the simulated-microgravity period) (2). TEE was calculated from Eq. 12 of the study of de Weir (6).

$B E E$ and $R E E$. During the simulated-microgravity period, BEE was measured every morning shortly after awakening (i.e., after a 12-h fast). REE was measured every evening, 4$5 \mathrm{~h}$ after the evening meal. Measurements were taken over 45-min periods with a Medical Graphics Critical Care Monitor and a Canopy system (Medical Graphics, St. Paul, MN) for breath-by-breath analysis of $\mathrm{CO}_{2}$ production and $\mathrm{O}_{2}$ uptake. During these measurements, subjects remained in a darkened room and were instructed to remain awake and rest quietly during measurements.

Body composition. Body composition was determined by dual-energy X-ray absorptiometry with a model DR $1,000 / \mathrm{W}$ whole body densitometer (Hologic, Waltham, MA) once 4 days before the bed-rest period and once 1 day after bed rest. All scans were completed in the whole body pencil-beam mode, and subjects were positioned according to the manufacturer's guidelines. To minimize interoperator differences as a source of error, one operator completed all scans and scan analyses. Subjects were scanned at approximately the same time of day on both occasions. Scans were analyzed with Hologic's routine whole body analysis software (version 5.35 ). The precision of the whole body scan in our laboratory is $0.6 \%$.

Statistical analyses. Average daily TEE was compared between free-living and simulated-microgravity conditions, and TEE was compared with energy intake during simulated microgravity. Body composition was compared before 
TABLE 1. Average daily TEE, REE, and BEE and energy intake in 9 healthy men during 10 days of head-down bed rest

\begin{tabular}{ccccc}
\hline \hline & $\begin{array}{c}\text { Ambulatory TEE, } \\
\text { kcal/day }\end{array}$ & \multicolumn{2}{c}{ Bed Rest, kcal/day } \\
\cline { 3 - 5 } & 2,928 & TEE & Energy intake & REE (P.M.) \\
\hline & 2,442 & 1,873 & $2,175 \pm 300$ & $1,739 \pm 141$ \\
2 & 2,493 & 2,432 & $2,772 \pm 474$ & $1,660 \pm 147$ \\
3 & 2,160 & 1,784 & $2,238 \pm 483$ & $1,843 \pm 205$ \\
4 & 3,111 & 1,914 & $2,359 \pm 500$ & $1,971 \pm 142$ \\
5 & 2,225 & 2,021 & $2,936 \pm 585$ & $2,102 \pm 151$ \\
6 & 3,414 & 1,886 & $2,895 \pm 637$ & $2,094 \pm 90$ \\
7 & 2,421 & 2,045 & $2,295 \pm 373$ & $1,719 \pm 166$ \\
8 & 2,579 & 2,300 & $2,839 \pm 363$ & $1,755 \pm 36 \pm 133$ \\
9 & $2,614 \pm 421$ & 2,052 & $2,866 \pm 565$ & $2,058 \pm 116$ \\
Mean \pm SD & $2,034 \pm 211^{*}$ & $2,597 \pm 320$ & $1,842 \pm 221$ \\
\hline
\end{tabular}

Values are means \pm SD where indicated. Total energy expenditure (TEE) was measured with doubly labeled water technique (see text), and energy intake was calculated from weighed food records. REE and BEE, resting and basal energy expenditures, respectively. Significantly less $(P<0.05)$ compared with * ambulatory TEE and energy intake during bed rest (analysis of variance); $†$ REE (paired Student's $t$-test).

and after the bed-rest period. Differences between the freeliving and head-down bed-rest conditions and those before and after the bed-rest period were tested with a paired Student's $t$-test. Daily changes in body weight, relative fluid balance (output/intake), energy intake, REE, and BEE during simulated microgravity were analyzed by repeated-measures analysis of variance with the Student-Newman-Keuls method of multiple comparisons between pairs (SigmaStat, Jandel Scientific, San Rafael, CA).

\section{RESULTS}

TEE was significantly less during simulated microgravity than under free-living conditions and was also less than energy intake during simulated microgravity (Table 1). Body weights, however, were no different before vs. during bed rest $(P>0.05)$ (Table 2). Fluid intake and urine output also remained unchanged during the 10-day bed-rest period (Fig. 2), indicating that no diuresis took place. This finding is supported by values of total body water calculated from ${ }^{18} \mathrm{O}$ dilution, which did not change between the ambulatory phase and the third day of bed rest. BEE remained unchanged during bed rest (Fig. 2) and was significantly less than REE (measured the evening after a 4- to 5-h fast) (Table 1).

TABLE 2. Change in physical characteristics before and after 10 days of head-down bed rest

\begin{tabular}{cccc}
\hline \hline Subject No. & Body Wt, kg & Body Fat, kg & Lean Body Mass, kg \\
\hline 1 & 3.10 & 1.88 & 1.21 \\
2 & -1.79 & -0.37 & -1.41 \\
3 & -0.30 & 0.81 & -1.10 \\
4 & -1.00 & 0.74 & -1.79 \\
5 & 1.20 & 0.30 & 0.98 \\
6 & -1.66 & 0.06 & -1.78 \\
7 & -0.74 & 0.26 & -0.99 \\
8 & 0.11 & 0.50 & -0.33 \\
9 & 0.07 & -0.24 & 0.27 \\
Mean \pm SD & $-0.11 \pm 1.52$ & $0.44 \pm 0.67 *$ & $-0.55 \pm 1.14$ \\
\hline
\end{tabular}

* Significantly greater after bed rest (paired Student's $t$-test), $P<0.05$.

\section{DISCUSSION}

In the absence of any change in BEE, the mean $21 \%$ reduction in TEE observed during head-down bed rest (simulated microgravity) implies that the reduction in physical activity associated with bed rest was responsible for reducing the energy requirements of the subjects during this period. Moreover, this is the first bed-rest study in which all three components of energy balance were measured; thus it is the first to allow measurements of intake and expenditure to be checked for internal consistency. In fact, using the difference between energy intake and energy expenditure to calculate the amount by which energy balance was positive yielded 5,630 kcal over the 10-day bed-rest period. This calculation is acceptably close to the value yielded by calculating average change in body-energy stores $(4,865 \mathrm{kcal})$ (assuming energy densities of $9,500 \mathrm{kcal} / \mathrm{kg}$ anhydrous fat mass and $1,000 \mathrm{kcal} / \mathrm{kg}$ fat-free mass).

Energy expenditure has been estimated during spaceflight by several means. For example, TEE for a group of cosmonauts was calculated from the amount of $\mathrm{CO}_{2}$ in the spacecraft atmosphere during the Soyuz-9 mission (3). The average energy expended by each crewmember (mean weight $80 \mathrm{~kg}$ ) during that 18-day mission was $2,300 \mathrm{kcal} /$ day (range $2,020-3,050 \mathrm{kcal} /$ day). TEE during the present microgravity simulation $(2,034$ $\mathrm{kcal} /$ day) was similar to energy expended during the first 6 days of the Soyuz- 9 mission $(2,090 \mathrm{kcal} /$ day $)$. However, as the cosmonauts adjusted to the flight environment and began to exercise, their energy expenditures (and thus their energy requirements) increased to $\sim 3,000 \mathrm{kcal} /$ day. These results suggest that the energy cost of physical activity varies in space (just as it does on Earth).

Results from the US Skylab missions, during which crewmembers kept detailed dietary records as part of metabolic-balance studies, showed progressive increases in available energy (intake minus fecal energy) during the missions that were matched by changes in daily exercise (13). The results from US and Russian space programs suggest that TEE may actually increase during spaceflight, depending on the level of physical activity, and that this fact must be considered 


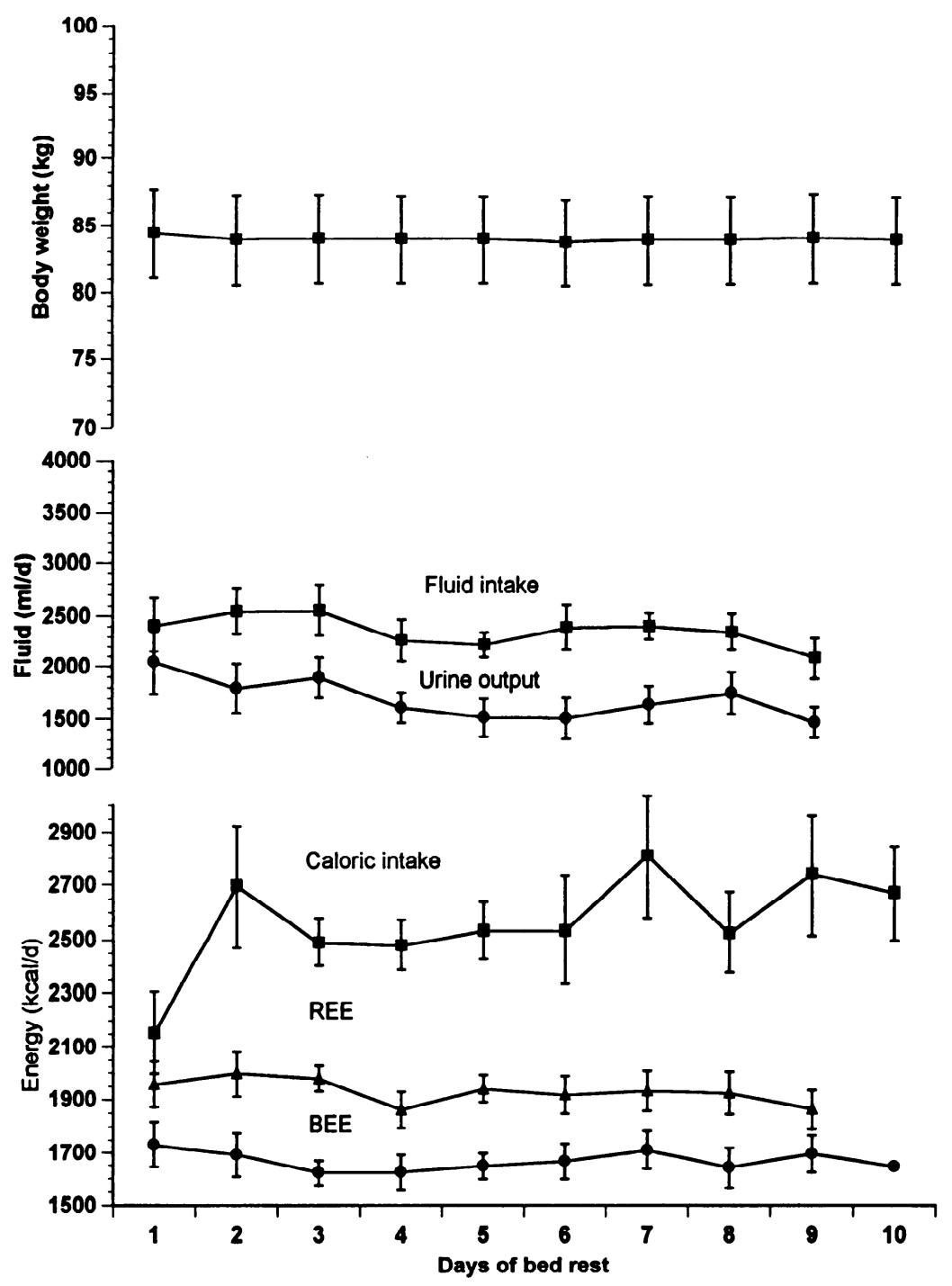

FIG. 2. Data are means $\pm \mathrm{SE}$ of body weight, fluid balance, energy intake, REE, and BEE in 9 healthy men during 10 days of $-6^{\circ}$ head-down bed rest.

in developing nutritional requirements for space. TEE under free-living conditions in our subjects was 2,641 $\mathrm{kcal} / \mathrm{day}$, which is at the lower end of the range of energy intake during the Skylab missions. Exercise was not controlled during our study, but most subjects reported that they did not exercise regularly.

All nine Skylab astronauts showed a small but consistent negative nitrogen balance, which suggests that spaceflight may induce or encourage the replacement of lean mass with fat (10). This view is consistent with results from previous bed-rest studies. Krebs et al. (9) showed that 5 wk of continuous bed rest significantly decreased lean body mass and increased body fat but did not affect total body weight. Our results were similar, except that the changes in body composition were less pronounced (i.e., lean body mass and body weight did not change, but percent body fat increased). Moreover, our subjects were in positive energy balance (Table 1), which is not typical of astronauts in space. During spaceflights, food intake tends to be affected by space motion sickness, schedules that limit the time available for meal preparation and consumption, and a diet of limited variety, all of which combine to produce weight loss in space. The replacement of lean mass with fat during bed rest suggests that body weight itself does not accurately reflect changes in energy balance. Thus, microgravity simulations addressing energy balance must rely on accurate measurements of body composition as well as body weight. Likewise, accurate means of measuring body composition during spaceflight are needed to qualify changes in body mass in space.

Under simulated microgravity conditions, our subjects showed no changes in BEE or REE during the 10day bed-rest period (Table 1). BEE estimated from the Harris-Benedict equation (8) was correlated with actual measurements in this study $(r=0.87, P<0.05)$. However, the Harris-Benedict equation resulted in overestimating the measured $\mathrm{BEE}$ value by $11.73 \pm$ $3.71 \%$, a statistically significant difference $(P<0.05)$. These results are similar to those found by the study of Daly et al. (5), conducted at Emory University and the Sloan-Kettering Cancer Center, in which the Harris-Benedict equation overpredicted BEE by $10.4 \pm$ 11.7 and $14.1 \pm 12.6 \%$, respectively. In addition, the average measured $\mathrm{BEE}$ was within $3 \%$ of that predicted when the more current equation by Cunningham 
(4) is used. These findings, in combination with the lack of change in BEE during the 10-day test period (Fig. 2), confirm that simulated microgravity had little if any effect on BEE. The evening measurements of REE were not significantly different from the HarrisBenedict estimates, with which they were also correlated $(r=0.80)$.

No changes in fluid status were noted during the 10-day bed-rest period. Fluid output as percentage of intake did not indicate diuresis and remained unchanged throughout the measurement period (Fig. 2). Also, estimates of total body water from the ${ }^{18} \mathrm{O}$ dilution were no different between the ambulatory phase and the third day of bed rest (Table 2). Our subjects remained well hydrated during the study, which is not typical of astronauts, at least during the first few days of flight. Space motion sickness, symptoms of which range from mild stomach awareness to severe nausea and vomiting, can cause dehydration and reduce fluid intake during the first few days of flight, which may account for some of the changes in total body water noted during spaceflight.

Summary. This study shows that TEE is reduced under conditions of head-down bed rest in which subjects are sedentary. The lack of change in BEE and REE during the bed-rest period suggests that lack of physical activity was responsible for reducing TEE in this study. These TEE results are similar to those noted during sedentary periods in spaceflight (3). However, the limited flight data available suggest that after cosmonauts or astronauts adjust to the flight environment, their physical activity levels and energy requirements increase to equal or exceed those on Earth (3, 11). Moreover, the consumption of an ad libitum diet during 10 days of bed rest increased the proportion of body fat without changing body weight in this study, suggesting that body weight itself does not accurately reflect energy balance during simulated microgravity. Finally, our subjects remained well hydrated and showed no evidence of diuresis during the 10-day bedrest period.

The authors gratefully acknowledge the efforts of Jeannie Nillen, Janis Davis-Street, and Christine Wogan of KRUG Life Sciences and Richard Socki of Lockheed Engineering and Sciences.

Address for reprint requests: R. J. Gretebeck, Universities Space Research Association, Biomedical Operations and Research Branch, Mail Code SD4, NASA-Johnson Space Center, Houston, TX 77058.

Received 29 September 1994; accepted in final form 13 February 1995.

\section{REFERENCES}

1. Berry, C. A. Summary of medical experience in the Apollo 7 through Apollo 11 manned space flights. Aerosp. Med. 41: 500,1970

2. Black, A. E., A. M. Prentice, and W. A. Coward. Use of food quotients to predict respiratory quotients for the doubly-labeled water method of measuring energy expenditure. Hum. Nutr. Clin. Nutr. 40: 381-391, 1986.

3. Chirkov, B. A. Energy expenditure of the crew during the eighteen-day flight of the "SOYUZ-9" spaceship. Kosm. Biol. Avia kosm. Med. 9: 48-51, 1975.

4. Cunningham, J. J. Body composition as a determinant of energy expenditure: a synthetic review and a proposed general prediction equation. Am. J. Clin. Nutr. 54: 963-969, 1991.

5. Daly, J. M., S. B. Heymsfield, C. A. Head, L. P. Harvey, D. W. Nixon, H. Katzeff, and G. D. Grossman. Human energy requirements: overestimation by widely used prediction equations. Am. J. Clin. Nutr. 42: 1170-1174, 1985.

6. De Weir, J. B. New methods for calculating metabolic rate with special reference to protein metabolism. J. Physiol. Lond. 109: $1-9,1949$.

7. Greenleaf, J. E. Energy and thermal regulation during bed rest and spaceflight. J. Appl. Physiol. 67: 507-516, 1989.

8. Harris, J. A., and F. G. Benedict. A Biometric Study of Basal Metabolism in Man. Washington, DC: Carnegie Institution, 1919. (Publication No. 279)

9. Krebs, J. M., V. S. Schneider, H. Evans, M. C. Kuo, and A. D. LeBlanc. Energy absorption, lean body mass, and total body fat changes during 5 weeks of continuous bed rest. Aviat. Space Environ. Med. 61: 314-318, 1990.

10. Lane, H. W. Nutritional questions relevant to space flight. Annu. Rev. Nutr. 12: 257-278, 1992.

11. Lane, H. W. Energy requirements for space flight. J. Nutr. 122: $13-18,1992$.

12. Leonard, J. I, C. S. Leach, and P. C. Rambaut. Quantitation of tissue loss during prolonged space flight. Am. J. Clin. Nutr. 43: $667-679,1983$.

13. Michel, E. L, J. A. Rummel, C. F. Sawin, M. C. Buderer, and J. D. Lem. Results of skylab medical experiment M171metabolic activity. In: Biomedical Results from Skylab (NASA $S P$-377), edited by R. S. Johnson and L. F. Dietlein. Washington, DC: NASA, p. 373-387, 1977.

15. Rambaut, P. C., M. C. Smith, and C. S. Leach. Nutrition and responses to zero gravity. Federation Proc. 36: 1678-1682, 1977.

16. Ravussin, E., S. Lillioja, and T. E. Anderson. Determinants of 24-hour energy expenditure in man: methods and results using a respiratory chamber. J. Clin. Invest. 78: 1568-1578, 1986.

17. Schoeller, D. A., E. Ravussin, Y. Shutz, K. Acheson, P. Baertschi, and E. Jequier. Energy expenditure by doubly labeled water: validation in humans and proposed calculation. Am. J. Physiol. 250 (Regulatory Intregrative Comp. Physiol. 19): R823 - R830, 1986.

18. Socki, R. A., H. R. Karlsson, and E. K. Gibson. Extraction technique for the determination of oxygen-18 in water using preevacuated glass vials. Anal. Chem. 64: 829-831, 1992.

19. Wong, W. W., L. S. Lee, and P. D. Klein. Deuterium and oxygen-18 measurements on microliter samples of urine, plasma, saliva, and human milk. Am. J. Clin. Nutr. 45: 905-913, 1987. 